\title{
Comparison of Alemtuzumab and Anti-thymocyte Globulin Treatment for Acute Kidney Allograft Rejection
}

OPEN ACCESS

Edited by:

Oriol Bestard,

Hospital Universitario de Bellvitge, Spain

Reviewed by:

Lionel Couzi,

Université de Bordeaux, France

Joshua Augustine,

Cleveland Clinic, United States

Farsad Alexander Eskandary,

Medical University of Vienna, Austria

${ }^{*}$ Correspondence:

Marieke van der Zwan

m.vanderzwan@erasmusmc.nl

TORCID:

Marieke van der Zwan orcid.org/0000-0002-9404-6135

Specialty section:

This article was submitted to Alloimmunity and Transplantation,

a section of the journal

Frontiers in Immunology

Received: 09 February 2020 Accepted: 26 May 2020

Published: 03 July 2020

Citation:

van der Zwan M, Clahsen-Van

Groningen MC, van den Hoogen MWF, Kho MML, Roodnat Jl, Mauff KAL, Roelen DL, van Agteren M, Baan CC and Hesselink DA (2020)

Comparison of Alemtuzumab and Anti-thymocyte Globulin Treatment for

Acute Kidney Allograft Rejection.

Front. Immunol. 11:1332.

doi: 10.3389/fimmu.2020.01332

\begin{abstract}
Marieke van der Zwan ${ }^{1,2 * t}$, Marian C. Clahsen-Van Groningen ${ }^{2,3}$, Martijn W. F. van den Hoogen ${ }^{1,2}$, Marcia M. L. Kho ${ }^{1,2}$, Joke I. Roodnat ${ }^{1,2}$, Katya A. L. Mauff ${ }^{4}$, Dave L. Roelen ${ }^{5}$, Madelon van Agteren ${ }^{1,2}$, Carla C. Baan ${ }^{1,2}$ and Dennis A. Hesselink ${ }^{1,2}$
\end{abstract}

${ }^{1}$ Division of Nephrology and Transplantation, Department of Internal Medicine, Erasmus MC, University Medical Center, Rotterdam, Netherlands, ${ }^{2}$ Rotterdam Transplant Group, Erasmus MC, University Medical Center, Rotterdam, Netherlands, ${ }^{3}$ Department of Pathology, Erasmus MC, University Medical Center, Rotterdam, Netherlands, ${ }^{4}$ Department of Biostatistics, Erasmus MC, University Medical Center, Rotterdam, Netherlands, ${ }^{5}$ Department of Immunohematology and Blood Transfusion, Leiden University Medical Center, Leiden, Netherlands

Rabbit anti-thymocyte globulin ( $r$ ATG) is currently the treatment of choice for glucocorticoid-resistant, recurrent, or severe acute allograft rejection (AR). However, rATG is associated with severe infusion-related side effects. Alemtuzumab is incidentally given to kidney transplant recipients as treatment for AR. In the current study, the outcomes of patients treated with alemtuzumab for AR were compared with that of patients treated with rATG for AR. The patient-, allograft-, and infection-free survival and adverse events of 116 alemtuzumab-treated patients were compared with those of 108 patients treated with rATG for AR. Propensity scores were used to control for differences between the two groups. Patient- and allograft survival of patients treated with either alemtuzumab or rATG were not different [hazard ratio (HR) 1.14, 95\%-confidence interval (Cl) 0.48-2.69, $p=0.77$, and $\mathrm{HR} 0.82,95 \%-\mathrm{Cl} 0.45-1.5, p=0.52$, respectively). Infection-free survival after alemtuzumab treatment was superior compared with that of rATG-treated patients ( $\mathrm{HR}$ 0.41, 95\%-Cl 0.25-0.68, $p<0.002$ ). Infusion-related adverse events occurred less frequently after alemtuzumab treatment. Alemtuzumab therapy may therefore be an alternative therapy for glucocorticoid-resistant, recurrent, or severe acute kidney transplant rejection.

Keywords: alemtuzumab, allograft rejection, rabbit anti-thymocyte globulin, kidney transplantation, $\mathrm{T}$ cell depletion

\section{INTRODUCTION}

Alemtuzumab is incidentally used to treat acute kidney allograft rejection (AR) (1-5). Alemtuzumab is a humanized monoclonal rat antibody directed against the cell surface glycoprotein CD52 (6). Treatment with alemtuzumab causes a long-lasting depletion of various cells of the adaptive ( $\mathrm{T}$ - and B cells) and innate immune system (NK cells, dendritic cells, monocytes, and granulocytes) (6). The drug is registered for the treatment of relapsing-remitting multiple sclerosis (7). The Campath ${ }^{\circledR}$ Distribution Program offers off-label treatment with alemtuzumab for other indications, including therapy for kidney transplant recipients and patients with chronic lymphocytic leukemia (8). 
Currently, rabbit anti-thymocyte globulin (rATG) is the treatment of choice for glucocorticoid-resistant, recurrent or severe (Banff grade IIA or higher) acute $\mathrm{T}$ cell-mediated rejection (aTCMR) (9). Although effective, rATG has several limitations, for instance infusion-related side effects (10-12). Alemtuzumab might be an alternative T cell-depleting therapy for AR with fewer infusion-related side effects (1-5).

The outcomes of alemtuzumab therapy for AR in kidney transplant recipients have only been reported in five small case series (with a cumulative number of 88 patients), concluding that patients with AR responded well to therapy with alemtuzumab (1-5). However, in only one of these reports, alemtuzumab was compared to rATG therapy and none of them were randomized controlled trials (1). Our center participated in one of these case series (1). In this case series, 11 patients with $A R$ and a contra-indication for rATG were treated with alemtuzumab. The incidence of the composite endpoint "treatment failure" was comparable between both groups (alemtuzumab 27\% vs. rATG $40 \%, p=0.89)$ and treatment with alemtuzumab was associated with fewer infusion related side effects and reduced costs (1).

Since 2012 and after our initial positive experience with alemtuzumab, it became the treatment of choice for all patients with glucocorticoid-resistant, severe or recurrent $\mathrm{AR}$ in the Erasmus MC (1). Here, we present further data on patientand allograft outcome on subsequent patients treated with alemtuzumab for AR in our center. Factors that influenced allograft survival were investigated, and we focused on the occurrence of infections, malignancies and autoimmune diseases. Patient-, allograft-, and infection-free survival of alemtuzumabtreated patients were compared with those of patients treated with rATG for AR (10).

\section{MATERIALS AND METHODS}

\section{Study Design}

A retrospective analysis was performed on data of kidney transplant recipients who were treated in the Erasmus MC, University Medical Center Rotterdam, with alemtuzumab (Campath ${ }^{\circledR}$, Sanofi Genzyme, United States) because of AR between January 2012 and January 2018. The study was approved by the medical ethical review board of the Erasmus MC (number 2018-1430). The patients were identified by the electronic medication prescription system of our hospital pharmacy. Patients with blood group AB0-incompatible kidney transplantations were excluded from the analysis, because they receive alemtuzumab as induction therapy (13).

The outcomes were compared to those of a cohort of patients treated with rATG (Thymoglobulin ${ }^{\circledR}$, Sanofi Genzyme,

\footnotetext{
Abbreviations: aABMR, Acute antibody-mediated rejection; aTCMR, Acute $\mathrm{T}$ cell-mediated rejection; AR, Acute kidney allograft rejection; C0, Pre-dose concentrations; CI, Confidence interval; CKD, Chronic kidney disease; CKDEPI, Chronic kidney disease Epidemiology Collaboration; CMV, Cytomegalovirus; CsA, Cyclosporine A; DGF, Delayed graft function; DSA, Donor-specific anti-HLA antibodies; EBV, Epstein-Barr virus; eGFR, Estimated glomerular filtration rate; HR, Hazard ratio; IQR, Interquartile range; IVIg, Intravenous immunoglobulins; MMF, Mycophenolate mofetil; PRA, Panel reactive antibodies; PNF, Primary non-function; rATG, Rabbit anti-thymocyte globulin.
}

United States) for AR between January 2002 and January 2012. The characteristics and outcomes of this cohort were described in detail previously (10).

All AR episodes (including recurrent AR) were biopsyproven and biopsies were re-evaluated according to the Banff 2015 (for rATG-treated patients) and Banff 2017 classification (for alemtuzumab-treated patients) by one dedicated renalpathologist (M.C.C-v.G.) (14-16). The presence of donorspecific anti-HLA antibodies (DSA) and non-donor-specific HLA antibodies against HLA-A, HLA-B, HLA-DR, and HLA-DQ were examined in alemtuzumab-treated patients using the singleantigen bead Luminex assay on serum samples collected at the time of AR. DSA directed against Cw and DP HLA molecules were not tested. The presence of DSA was not routinely tested in the period 2002-2012 when rATG still was the therapy of choice (10). Therefore, the biopsies of the rATG-treated patients could not be reclassified according to the Banff 2017 criteria (14).

Of patients treated with alemtuzumab, patient survival, allograft function [estimated glomerular filtration rate (eGFR); Chronic Kidney Disease Epidemiology Collaboration (CKD-EPI) (17)], allograft survival (censored for death), variables that could influence allograft survival (patient and donor characteristics, type of immunosuppressive therapy, and type and grade of rejection), and adverse events were assessed. Baseline eGFR was defined as the highest eGFR in the 3 months prior to AR. Delayed graft function (DGF) was defined as the need for dialysis in the first week after transplantation. Allograft loss was defined as the need for dialysis or retransplantation. The follow-up period for allograft loss and infection was from the day of $\mathrm{T}$ cell-depleting therapy until death, retransplantation, or loss to follow-up. Malignancies and mortality were evaluated until the last follow-up visit, which could be after subsequent kidney transplantation. The Dutch national pathology archive PALGA (Pathologisch-Anatomisch Landelijk Geautomatiseerd Archief, https://www.palga.nl/) was used for collecting of data relating to the occurrence of malignancy. Infections were considered serious if the infection necessitated hospitalization or occurred during hospital admission for another reason.

The allograft- and patient survival data of patients who had received a kidney transplant in the same time periods in our center and were not treated with $\mathrm{T}$ cell-depleting therapy was also compared to the patients treated with $\mathrm{T}$ cell-depleting therapy for AR.

\section{Maintenance Immunosuppressive Therapy}

The standard immunosuppressive regimen included induction therapy with basiliximab (Simulect ${ }^{\circledR}$, Novartis Pharma, Basel, Switzerland) $20 \mathrm{mg}$ intravenously on days 0 and 4 after transplantation, followed by maintenance therapy with tacrolimus (Prograf ${ }^{\circledR}$, Astellas Pharma, Leiden, the Netherlands), mycophenolate mofetil (MMF; Cellcept ${ }^{\circledR}$, Roche Pharmaceuticals, Basel, Switzerland) and glucocorticoids.

Basiliximab became part of our standard immunosuppressive regimen in 2009. Dosing of tacrolimus and MMF was based on pre-dose concentrations $\left(\mathrm{C}_{0}\right)$. Target $\mathrm{C}_{0}$ for tacrolimus was, respectively, 10-15 $\mu \mathrm{g} / \mathrm{L}$ (weeks 1-2), 8-12 $\mu \mathrm{g} / \mathrm{L}$ (weeks 3-4), 5-10 $\mu \mathrm{g} / \mathrm{L}$ (weeks 5-12), and 4-8 $\mu \mathrm{g} / \mathrm{L}$ from month 4 onwards. 
MMF was started at $1,000 \mathrm{mg}$ twice daily and subsequent dosing was based on $\mathrm{C}_{0}$ (target $\mathrm{C}_{0}$ was $1.5-3.0 \mathrm{mg} / \mathrm{L}$ ). Glucocorticoids were given as an intravenous dose of $100 \mathrm{mg}$ on days $0-3$ and followed by a dose of $20 \mathrm{mg} /$ day (days $4-14$ ). Thereafter, glucocorticoids were tapered off and completely withdrawn around month 4 .

\section{Treatment of AR}

The first-line treatment of aTCMR was methylprednisolone $1,000 \mathrm{mg}$ (Solu-Medrol ${ }^{\circledR}$, Pfizer, New York, the United States) intravenously daily for 3 consecutive days, followed by a second-line treatment with alemtuzumab or rATG in case of a glucocorticoid-resistant, recurrent or severe aTCMR (Banff grade IIA or higher). rATG was administered as a single bolus [ $4 \mathrm{mg} / \mathrm{kg}$ (actual bodyweight, no maximum dose limit)] intravenously (10). Alemtuzumab was administered subcutaneously (18). The first 14 patients were treated with alemtuzumab $(30 \mathrm{mg})$ daily for 2 consecutive days. Since $\mathrm{T}$ cell-depletion already occurred after one dose of alemtuzumab, the next patients received a single dose $(30 \mathrm{mg})$. To prevent infusion-related side effects patients were premedicated with glucocorticoids (50 mg intravenously), acetaminophen (4 times daily $1,000 \mathrm{mg}$ ), and clemastine ( $4 \mathrm{mg}$ intravenously). The alemtuzumab-treated patients were discharged the same day if no severe side-effects were noted. T- and $\mathrm{B}$ cell counts were measured with $\mathrm{BD}$ FACSCanto ${ }^{\mathrm{TM}}$ software every 3 months until the $\mathrm{T}$ cell count was $>200 \times 10^{6} / \mathrm{L}$. In the patients treated with $\mathrm{rATG}$, a $\mathrm{CD}^{+}$ $\mathrm{T}$ cell count $<200 \times 10^{6} / \mathrm{L}$ was aimed for a duration of 2 weeks during which patients were hospitalized (10). If $\mathrm{CD}^{+} \mathrm{T}$ cell counts increased during this period, a repeat dose of rATG was administered. All patients received prophylaxis for Pneumocystis jirovecii (sulfamethoxazole/trimethoprim) and cytomegalovirus (CMV; valganciclovir, except for CMV seronegative recipients with CMV seronegative donors) until the $\mathrm{T}$ cell count was $>200 \times 10^{6} / \mathrm{L}$. Patients with aABMR or mixed type AR could additionally be treated with intravenous immunoglobulins (IVIg), plasma-exchange, or both according to the Kidney Disease: Improving Global Outcomes (KDIGO) guideline (9).

\section{Statistical Methods}

Categorical variables are presented as number (percentage). Continuous variables are presented as mean with standard deviation for normally distributed variables or median with interquartile range (IQR) for non-normally distributed variables. For differences between unpaired non-normally distributed continuous data or unpaired categorical data, the KruskalWallis and Mann-Whitney $U$-tests, and the Chi-squared and Fisher's exact tests were used. Kaplan-Meier survival analysis was used to examine subgroups (e.g., age categories and rejection types) within the alemtuzumab group and to compare allograftand patient survival between alemtuzumab-treated patients and patients transplanted in the same period and who were not treated with alemtuzumab.

The influence of predictor variables on allograft survival in alemtuzumab-treated patients was analyzed with multivariable Cox proportional hazard regression analysis. Due to the number of events (41 allograft losses), the number of variables that could be included per analysis was limited. The influence of the most significant variable was tested in the presence of all the other variables one by one, and the non-significant variables were eliminated from the model by backward elimination.

Propensity scores were used to control for baseline differences between the patients treated with rATG and alemtuzumab (19). They were acquired by performing a logistic regression with therapy type as the outcome variable. Covariates included in the logistic model were: age of the patient at time of AR, gender, primary kidney disease, donor type (living/deceased), induction therapy ( $43 \%$ of patients treated with $\mathrm{rATG}$ received induction therapy, vs. $97.3 \%$ of alemtuzumab treated patients), maintenance therapy, time to AR, and type of AR. The resulting propensity score was used as a covariate in Cox proportional hazards regression models (for calculation of the patient-, allograft-, and infection-free survival), in linear regression models (for continuous outcomes), and in logistic regression models (for categorical outcomes).

A two-sided $p<0.05$ was considered statistically significant. GraphPad Prism, version 5 (San Diego, CA, USA), SPSS version 21 (SPSS Inc., Chicago, IL, USA), and R (R Foundation for Statistical Computing, Vienna, Austria, version 3.5.1) were used for the statistical analysis.

\section{RESULTS}

\section{Patient Demographics}

Between January 2012 and January 2018, 1,214 patients received a kidney transplant at our center. Of these, 113 patients (9.3\%) were treated with alemtuzumab for AR. Three patients were treated with alemtuzumab twice because of two separate rejection episodes of the same kidney transplant. Between January 2002 and January 2012, 1,107 patients were transplanted with a kidney and 108 patients of these $(9.8 \%)$ were treated with rATG for AR (10). The median cumulative dose of rATG per patient was $7.4 \mathrm{mg} / \mathrm{kg}$. Baseline characteristics of patients treated with either alemtuzumab or rATG for AR are presented in Table 1.

Induction therapy with basiliximab was given to 303 (27.4\%) of all patients transplanted between 2002 and 2012 (the rATG period) and to $1,065(87.8 \%)$ of all patients between 2012 and 2018 (the alemtuzumab period). As a result, significantly more patients treated with alemtuzumab (93.8\%) had previously received basiliximab induction therapy compared to rATGtreated patients $[29.2 \% ; p<0.0001$ (Table 2)]. A tacrolimusand MMF-based maintenance therapy was given to $81 \%$ of alemtuzumab-treated patients and to $72.2 \%$ of rATG-treated patients $(p=0.08$; Table 2). First line therapy for AR was methylprednisolone in $94.8 \%$ of alemtuzumab-treated patients, and to $86.1 \%$ or rATG-treated patients (Table 2).

Sixty-four alemtuzumab-treated patients (55.2\%) and 64 (59.3\%) rATG-treated patients had an early AR (within 3 months after transplantation; Table 3). The distribution of the Banff grade of AR was not different between the patients treated with alemtuzumab or rATG ( $p=0.89$; Table 3$)$. In 18 patients $(15.5 \%)$, a second kidney allograft biopsy was performed after the initial treatment with methylprednisolone and immediately before alemtuzumab treatment to confirm ongoing AR (Table S1). 
TABLE 1 | Baseline characteristics of patients treated with either alemtuzumab or rATG.

\begin{tabular}{lccc}
\hline Characteristic & $\begin{array}{c}\text { Alemtuzumab } \\
(\boldsymbol{n}=\mathbf{1 1 3})\end{array}$ & rATG (n=108) & $\boldsymbol{p}$-value \\
\hline $\begin{array}{l}\text { Recipient age at } \\
\text { transplantation-yr. }\end{array}$ & $56(39-63)$ & $45(34-55)$ & 0.0001 \\
$\begin{array}{l}\text { Recipient age at } \\
\text { rejection-yr. }\end{array}$ & $56(40-63)$ & $46(35-56)$ & 0.0002 \\
$\begin{array}{l}\text { Donor age-yr. } \\
\text { Female sex-no. (\%) }\end{array}$ & $54(43-63)$ & $54(46-61)$ & 0.82 \\
\hline
\end{tabular}

Cause of ESRD-no.

$\begin{array}{llll}\text { DM/HTN/GN/PKD/ 26/22/21/9/7/27/4 23/10/18/16/17/16/3 } & 0.04\end{array}$

Ethnic distribution-no.

Caucasian/Black/
Asian/Arab/other
Transplant number-no.
1/2/3

$$
79 / 17 / 8 / 11 / 1 \quad 70 / 16 / 5 / 5 / 7
$$

Preemptive kidney

transplantation-no. (\%)

Donor type-no.

\section{LR/LUR/DBD/DCD}

HLA mismatch

HLA A: 0/1/2

HLA DR: 0/1/2

PRA actual-no. (\%)

$0-5 \%$
$6-83 \%$

\section{4-100\%}

PRA peak-no. (\%)

$\begin{array}{lcc}0-5 \% & 69(59.5) & 62(59) \\ 6-83 \% & 31(26.7) & 32(30.5) \\ 84-100 \% & 16(13.8) & 11(9.5)\end{array}$

CMV IgG serostatus

recipient-no. (\%)

Positive

83 (73.6)

75 (70.8)

0.76

EBV IgG serostatus

recipient-no. (\%)

$$
\text { Positive }
$$

$88 / 22 / 6$
$41(35.3)$

$76 / 25 / 5$

25 (23.4)

$$
0.71
$$$$
0.06
$$

\section{7/55/12/22}

$35 / 47 / 15 / 10$

0.12

$\begin{array}{lll}26 / 61 / 29 & 21 / 60 / 23 & 0.75 \\ 10 / 53 / 53 & 11 / 55 / 38 & 0.39 \\ 21 / 55 / 40 & 13 / 50 / 41 & 0.48 \\ & & 0.52\end{array}$

$\begin{array}{cc}93(80.2) & 81(77.1) \\ 22(19.0) & 21(20) \\ 1(0.8) & 3(2.9)\end{array}$

Data are numbers (\%) or median (interquartile range). *Other kidney diseases included focal segmental glomerulosclerosis, vascular disease, septic shock, kidney dysplasia/nephrectomy, congenital nephrotic syndrome, Alport syndrome, nephronophtisis, drug intoxication, RCAD syndrome, or tubulointerstitial nephritis. Data of rATG-treated patients are prescribed previously (10). CMV, cytomegalovirus; DBD, donation after brain death; $D C D$, donation after circulatory death; DM, diabetes mellitus; EBV, Epstein-Barr virus; ESRD, end stage renal disease; GN, glomerulonephritis; HLA, human leucocyte antigen; HTN, hypertensive nephropathy; $L R$, living related; $L U R$, living unrelated; PKD, polycystic kidney disease; PRA, panel reactive antibody; rATG, rabbit anti-thymocyte globulin.

\section{Patient Survival}

Patient survival of patients treated with either alemtuzumab or rATG for AR is depicted in Figure 1A. Compared with the historical rATG cohort, the patient survival
TABLE 2 | Immunosuppressive therapy in patients treated with alemtuzumab or rATG.

\begin{tabular}{lccc}
\hline $\begin{array}{l}\text { Immunosuppressive } \\
\text { therapy }\end{array}$ & $\begin{array}{c}\text { Alemtuzumab } \\
(\boldsymbol{n}=\mathbf{1 1 3})\end{array}$ & rATG $(\boldsymbol{n}=\mathbf{1 0 8})$ & $\boldsymbol{p}$-value \\
\hline $\begin{array}{l}\text { Induction } \\
\text { therapy-no. (\%) }\end{array}$ & & $<0.0001$ \\
None & $3(2.7)^{\star}$ & $62(57.4)$ & \\
Basiliximab & $106(93.8)$ & $33(29.2)$ & \\
rATG & $2(1.8)$ & $10(8.8)$ & \\
Rituximab & $2(1.8)$ & $0(0)$ & \\
Daclizumab & $0(0)$ & $2(1.9)$ & 0.08 \\
Maintenance & & &
\end{tabular}

\begin{tabular}{|c|c|c|c|}
\hline TAC/MMF/glucocorticoids & 78 (67.2) & $58(53.7)$ & \\
\hline TAC/MMF & $16(13.8)$ & $20(18.5)$ & \\
\hline $\begin{array}{l}\text { TAC + other } \\
\text { (non-MMF) }\end{array}$ & $11(9.5)$ & $6(5.6)$ & \\
\hline $\begin{array}{l}\text { MMF + other } \\
\text { (non-TAC) }\end{array}$ & $11(9.5)$ & $20(18.5)$ & \\
\hline \multicolumn{4}{|l|}{$\begin{array}{l}\text { Anti-rejection } \\
\text { herapy-no. (\%) }\end{array}$} \\
\hline $\begin{array}{l}\text { Methylprednisolone } \\
\text { prior to T cell-depleting } \\
\text { therapy }\end{array}$ & $110(94.8)$ & $93(86.1)$ & 0.04 \\
\hline $\begin{array}{l}\text { Cumulative dose of } \\
\text { methylprednisolone, } \\
\text { mg }\end{array}$ & & & 0.004 \\
\hline 1,000 & $0(0)$ & $2(2.2)$ & \\
\hline 2,000 & $1(0.9)$ & $9(8.2)$ & \\
\hline 3,000 & $96(87.3)$ & 79 (71.8) & \\
\hline 4,000 & $1(0.9)$ & $0(0)$ & \\
\hline 6,000 & $12(10.9)$ & $3(2.7)$ & \\
\hline
\end{tabular}

immunosuppression-

$$
\text { no. (\%) }
$$

Additional anti-rejection

therapy in patients with

ABMR

Intravenous
immunoglobulins
Plasma-exchange +
intravenous
immunoglobulins
Additional
anti-rejection therapy
in patients with
mixed AR
Intravenous
immunoglobulins
Plasma-exchange +
intravenous
immunoglobulins

Data are numbers (\%). *In three patients no induction therapy was administered because of an HLA-identical donor. TAC + other regime contained combinations of TAC, glucocorticoids, everolimus, or azathioprine. Other combinations existed of a combination of azathioprine, glucocorticoids, everolimus, cyclosporine A, AEB071, or FTY720. MMF + other regime contained combinations of MMF, glucocorticoids, cyclosporine $A$, everolimus, or belatacept. Data of rATG-treated patients are prescribed previously (10). MMF, mycophenolate mofetil; TAC, tacrolimus.

of the alemtuzumab group was not different [hazard ratio (HR) 1.14 , 95\%-confidence interval (CI) $0.48-$ 2.69, $p=0.77 ;$ Figure 1A), also when only those patients who were treated with basiliximab induction 
TABLE 3 | Rejection characteristics.

\begin{tabular}{|c|c|c|c|}
\hline $\begin{array}{l}\text { Rejection } \\
\text { characteristic }\end{array}$ & $\begin{array}{l}\text { Alemtuzumab } \\
\qquad(n=116)^{\$}\end{array}$ & $\begin{array}{c}\text { rATG } \\
(n=108)\end{array}$ & $p$-value \\
\hline Time to rejection-days & $32(2-1644)$ & $24(8-339)$ & 0.83 \\
\hline $\begin{array}{l}\text { Early rejection (<3 } \\
\text { months)-no. (\%) }\end{array}$ & $64(55.2)$ & $64(59.3)$ & 0.59 \\
\hline $\begin{array}{l}\text { Late rejection (>3 } \\
\text { months)-no. (\%) }\end{array}$ & $52(44.8)$ & $44(40.7)$ & 0.59 \\
\hline $\begin{array}{l}\text { Delayed graft function } \\
\text { during AR }\end{array}$ & $33(28)$ & $19(17.6)$ & 0.06 \\
\hline Banff classification-no. ${ }^{*}$ & & & 0.89 \\
\hline \multicolumn{4}{|l|}{ aTCMR } \\
\hline aTCMR IA/BB & $1 / 9$ & $6 / 8$ & \\
\hline aTCMR \|A/IB/III & 29/23/2 & 21/20/1 & \\
\hline Borderline aTCMR & 3 & 0 & \\
\hline \multicolumn{4}{|l|}{ ABMR } \\
\hline aABMR & 17 & $12^{@}$ & \\
\hline $\mathrm{DSA}+$ and $\mathrm{C} 4 \mathrm{~d}+$ & 7 & & \\
\hline DSA+ and C4d- & 0 & & \\
\hline DSA- and C4d+ & 7 & & \\
\hline $\begin{array}{l}\text { C4d+, no DSA } \\
\text { tested }\end{array}$ & 2 & & \\
\hline $\begin{array}{l}\text { Histologic features } \\
\text { of ABMR, no } \\
\text { DSA/C4d }\end{array}$ & $1^{¥}$ & & \\
\hline c/aABMR & $1^{\S}$ & 3 & \\
\hline \multicolumn{4}{|l|}{$\begin{array}{l}\text { Mixed aTCMR with } \\
\text { aABMR }\end{array}$} \\
\hline aTCMR I/II/III & 9/7/2 & 8/10/0@ & \\
\hline $\mathrm{DSA}+$ and $\mathrm{C} 4 \mathrm{~d}+$ & 5 & & \\
\hline DSA+ and C4d- & 6 & & \\
\hline DSA- and C4d+ & 4 & & \\
\hline $\begin{array}{l}\text { C4d+, no DSA } \\
\text { tested }\end{array}$ & 3 & & \\
\hline $\begin{array}{l}\text { Mixed aTCMR with } \\
\text { c/aABMR }\end{array}$ & $1^{\$}$ & 1 & \\
\hline
\end{tabular}

Data are numbers (\%) or median (interquartile range). ${ }^{\$}$ A total of 113 patients were treated with alemtuzumab, however three patients were treated with alemtuzumab twice because of two separate rejection episodes of the same kidney transplant *Banff classification of aTCMR, ABMR and mixed AR were compared. Re-classification in 12 biopsies of alemtuzumab-treated patients and 18 biopsies of rATG-treated patients was not possible because the biopsies were missing from archives. The primary pathological diagnosis of these biopsies was aTCMR in five patients, ABMR in two patients, and mixed AR in five patients. Data of rATG-treated patients are prescribed previously (10). ${ }^{¥}$ Histologic features of ABMR with glomerulitis and peritubular capillaritis, but C4d staining was negative and no DSAs were present. ${ }^{\S}$ The patients with c/aABMR had no DSAs and C4d staining was positive in the peritubular capillaries. \$The patient with mixed c/aABMR had DSAs and C4d staining was negative. ${ }^{@} D S A$ s were not routinely measured in the rATGtreated patients. ABMR antibody mediated rejection; aABMR, active antibody mediated rejection; aTCMR, acute $T$ cell mediated rejection; c/aABMR, chronic/active antibody mediated rejection; DGF, Delayed graft function (need for dialysis in the first week after transplantation); DSA, de novo donor specific antibodies.

therapy were included (HR 1.74, 95\%-CI 0.68-4.46, $p=0.25$; Figure 2A).

The patient survival of alemtuzumab-treated patients was significantly lower compared with the patients transplanted in the same period and who were not treated with alemtuzumab
(HR 2.38, 95\%-CI 1.25-4.54, $p=0.0036$, Figure S1A). In the total follow-up period [median 2.8 years (IQR $1.3-3.8$ years)], 18 patients died after a median of 1.45 years (IQR 0.92-2.93; Table S2). A univariable Cox proportional hazard regression analysis was performed to investigate which variables influenced the risk of death in patients treated with alemtuzumab. The only variable that was associated with the risk of death was age of the recipient (HR per year 1.09, 95\%-CI 1.04-1.14, $p$ $<0.0001$; Table S3). This increased risk of death was seen in alemtuzumab-treated patients older than 50 years at the time of transplantation (Figure S2).

A comparison between the patient survival of rATG-treated and patients transplanted in the same period and who were not treated with rATG is shown in Figure S1C and was described previously (10).

\section{Kidney Allograft Survival}

Death-censored kidney allograft survival of alemtuzumabtreated patients was not different compared to that of patients who received $\mathrm{rATG}$ for AR (HR 0.82, 95\%-CI 0.45-1.50, $p=0.52$; Figure 1B). A similar survival was also observed when only those patients who were treated with basiliximab induction therapy were included (HR 1.10, 95\%-CI 0.57-2.10, $p=0.78$; Figure 2B). Additional information about the kidney allograft function after alemtuzumab or rATG therapy for AR is provided in Figure S3.

The allograft survival of alemtuzumab-treated patients was significantly worse compared to the allograft survival of patients that were transplanted in the same period and who were not treated with alemtuzumab (HR 258.0, 95\%-CI 112.0591.3, $p<0.0001$; Figure S1B). During the follow-up (median 2.2 years, IQR 1-3.5), 41 (35.3\%) patients lost their kidney allograft after alemtuzumab therapy for AR, of which six never had a functioning graft [primary non-function (PNF)]. To investigate which variables influenced allograft survival in alemtuzumab-treated patients, a Cox proportional hazard regression analysis was performed. In the univariable model, age of the recipient, number of HLA mismatches, glucocorticoid maintenance treatment, timing of $\mathrm{AR}$, and the $\Delta$ eGFR (percentage change between baseline eGFR and eGFR at the moment of AR) significantly influenced the risk for deathcensored allograft loss $(p<0.05$, Table S4) in alemtuzumabtreated patients. The variables glucocorticoid use and timing of rejection were related because all patients with an early acute rejection used glucocorticoids as maintenance therapy, while only $56.6 \%$ of patients with a late acute rejection used glucocorticoids $(p<0.0001)$. The Banff grade of rejection did not influence allograft survival $(p=0.19)$. Allograft survival of alemtuzumab-treated patients suffering from either aTCMR or aABMR is shown in Figure S4.

The final multivariable model showed that patients with actual panel reactive antibodies (PRA) $>6 \%$, and patients with a $\Delta$ eGFR of more than $50 \%$ had an inferior allograft survival after alemtuzumab therapy (Figure 3). Patients using glucocorticoids at time of $\mathrm{AR}$, and patients with more HLA mismatches, showed a superior allograft survival after alemtuzumab therapy (Figure 3). Several variables were compared between patients with an HLA mismatch of 0-3 and an HLA mismatch of 4-6 

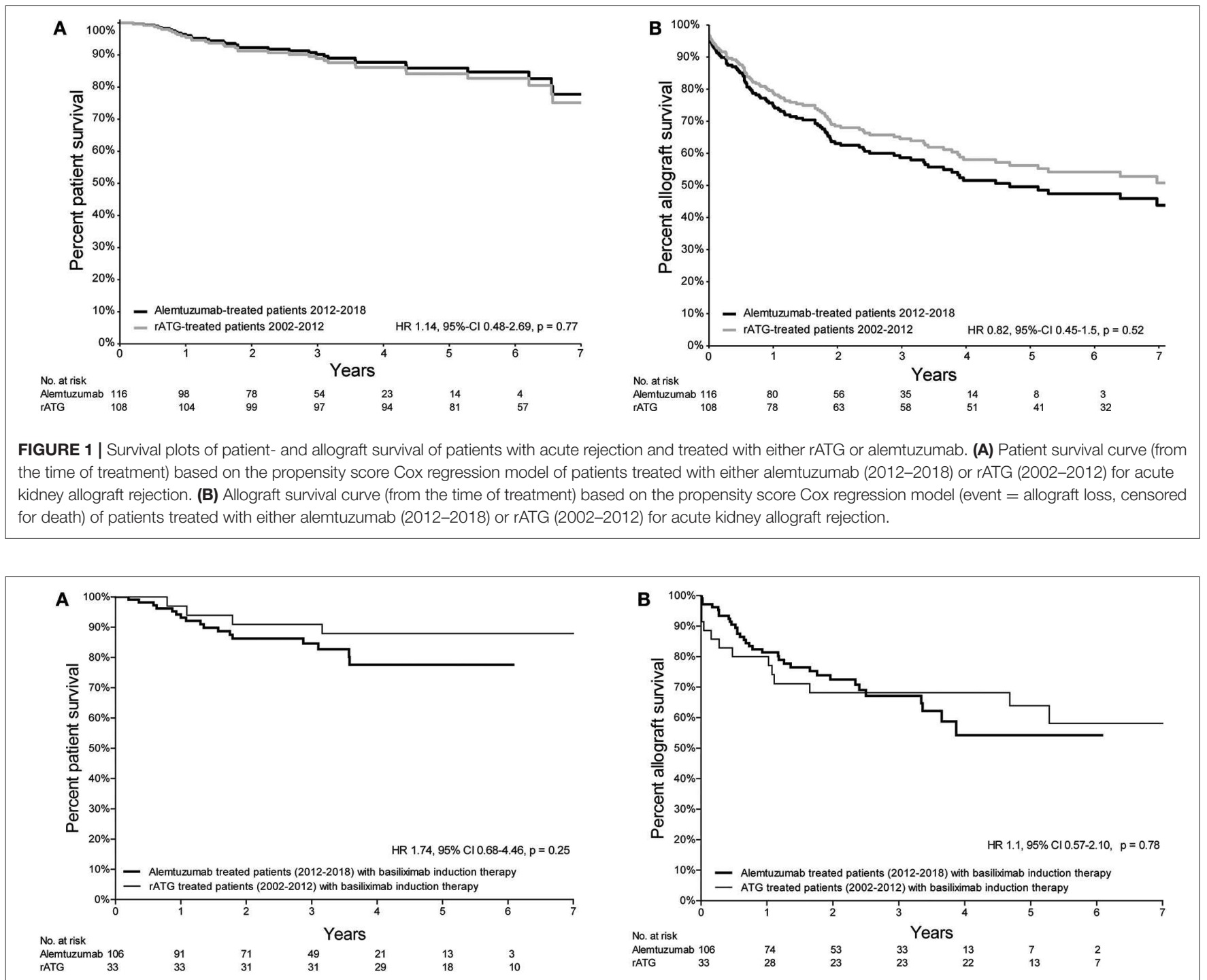

FIGURE 2 | Kaplan-Meier survival curves of the patient- and allograft survival of patients treated with basiliximab induction therapy. (A) Kaplan-Meier curve of the patient survival curve (from the time of treatment) of patients treated with either alemtuzumab (2012-2018) or rATG (2002-2012) for AR and who received induction therapy with basiliximab. (B) Kaplan-Meier curve of the allograft survival curve (from the time of treatment) of patients treated with either alemtuzumab (2012-2018) or rATG (2002-2012) for AR and who received induction therapy with basiliximab.

(Table S5). One variable was significantly different: $42(72 \%)$ recipients with 4-6 HLA mismatches received a living unrelated donor kidney (Table S5), while 13 (23\%) recipients with 03 HLA received a living unrelated donor kidney $(p<0.001$; Table S5).

The allograft survival of patients treated with survival of rATG was worse compared to that of patients who were not treated with rATG and transplanted in the same time period (HR 15.9, 95\%-CI 9.2-27.4, $p<0.0001$; Figure S1D). A multivariable Cox proportional hazard regression analysis was performed for patients treated with $\mathrm{rATG}$ for AR and reported previously (10). This analysis demonstrated that allograft survival was superior in patients with an early AR compared with a late AR (10).

\section{Adverse Events}

Infusion-related side effects also occurred less frequently in alemtuzumab-treated patients compared with the patients treated with rATG (Table 4). No alemtuzumab-treated patients experienced serum sickness vs. five patients in the rATG group $(p=0.02)$. No patients experienced cytokine release syndrome or pulmonary edema after alemtuzumab. The median duration of hospitalization was 3 days (IQR 1-6) in patients treated with alemtuzumab and 15 days (IQR 13-19) in rATG-treated patients.

The infection-free survival (excluding CMV, EBV, and BK virus infections) in the first year after alemtuzumab treatment was significantly better compared with the infection-free survival of the rATG-treated patients (HR 0.41, 95\%-CI 0.25-0.68, p $<0.002$; Figure 4). CMV reactivation occurred in 25 patients 


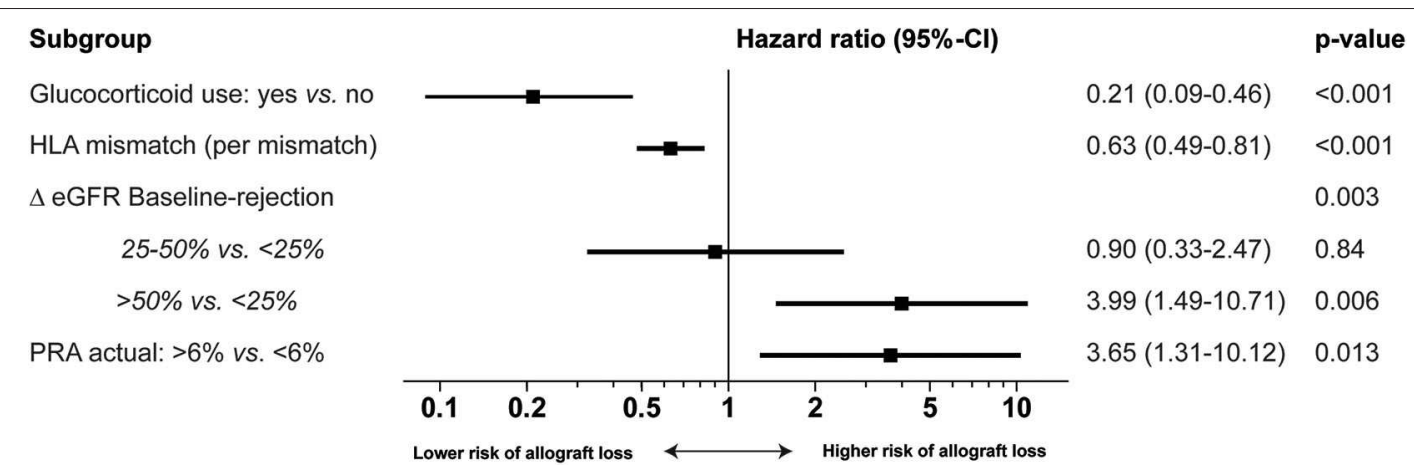

FIGURE 3 | Multivariable Cox proportional hazard regression analysis of risk for allograft loss in alemtuzumab-treated patients. Multivariable analysis of the risk of allograft loss with hazard ratio $[\operatorname{Exp}(\mathrm{B}), 95 \%$-confidence interval and $p$-value]. Delta $(\Delta)$ eGFR baseline-moment of rejection is the percentage change between the baseline eGFR and eGFR at the moment of rejection. Glucocorticoid use means maintenance therapy with glucocorticoids during the rejection. PRA Panel reactive antibodies.

TABLE 4 | Adverse events after therapy with alemtuzumab or rATG.

\begin{tabular}{lccc}
\hline Adverse events & Alemtuzumab & rATG & p-value \\
\hline $\begin{array}{l}\text { Fever*-no. (\%) } \\
\text { Systolic blood pressure } \\
<90 \text { mmHg-no. (\%) }\end{array}$ & $10(8 \%)$ & $42(61.8 \%)$ & $<0.001$ \\
$\begin{array}{l}\text { Tachycardia } \\
>100 / \text { min-no. (\%) }\end{array}$ & $18(15.5 \%)$ & $44(69.8 \%)$ & 0.003 \\
$\begin{array}{l}\text { Interventions-no. } \\
\text { Transfer to ICU }\end{array}$ & $1(0.9 \%)$ & $5(4.6 \%)$ & \\
$\begin{array}{l}\text { Supplemental } \\
\text { oxygen }\end{array}$ & $1(0.9 \%)$ & $9(13.4 \%)$ & 0.001 \\
Volume resuscitation & 0 & $6(9.0 \%)$ & 0.03 \\
\hline
\end{tabular}

Data are numbers (percentage) and median (interquartile range). Fever, blood pressure, tachycardia and interventions were registered in the $24 \mathrm{~h}$ after therapy. Under-reporting of the incidence of infusion-related adverse events is possible in 37 patients who were dismissed on the day of alemtuzumab therapy. Data of rATG-treated patients are prescribed previously (10). *Fever was defined as temperature above $38.5^{\circ} \mathrm{C}$.

$(21.6 \%)$ treated with alemtuzumab (Table S6), compared to 27 patients $(25 \%)$ in the rATG group $(p=0.10)$. In both the alemtuzumab- and rATG-treated groups, two patients experienced a primary CMV infection $(p=0.50)$. Additional information on the occurrence of infections is presented in Table S6.

Secondary autoimmune events have been described after administration of alemtuzumab (6). In the current study, two patients developed inflammatory polyneuropathy (one case of Guillain-Barre syndrome and one case of chronic inflammatory demyelinating polyneuropathy) after alemtuzumab treatment (20). No patients were diagnosed with autoimmune thyroid disorders, idiopathic thrombocytopenic purpura or autoimmune nephropathy.

Repopulation of $\mathrm{T}$ cells $>200 \times 10^{6} / \mathrm{L}$ occurred in $55.7 \%$ of alemtuzumab-treated patients in the first year after administration (Figures S5A,B). In $40.2 \%$ of the patients, repopulation of B cells $>100 \times 10^{6} / \mathrm{L}$ (Figures S5C,D) was seen at 1 year.
Solid tumors were diagnosed in seven alemtuzumab-treated patients during the total follow-up [median 2.8 years (IQR 1.33.8); Table S7]. The incidence of solid tumors was 2.3 per 100 person-years with a median time after alemtuzumab therapy of 28 months (IQR 9-38), and the age at the time of diagnosis was 65 years (IQR 60-76). Seven patients were diagnosed with skin cancer: 21 basal cell carcinomas and 12 squamous cell carcinomas. In the rATG treated patients, 14 malignancies were diagnosed after a mean time of 63 months (standard deviation 45 months) during the follow-up of 6.8 years (IQR 4.9-9.1; Table S8) (10).

\section{DISCUSSION}

In the current study, the largest cohort of patients treated with alemtuzumab for AR is described. The results of this study suggest that alemtuzumab could be an alternative to rATG for the treatment of glucocorticoid-resistant, severe or recurrent AR. Compared to rATG, allograft- and patient survival of patients treated with alemtuzumab was not different. Moreover, adverse events and infections seemed to occur less frequently in patients treated with alemtuzumab compared with rATG-treated patients.

Alemtuzumab seems as effective as rATG for the prevention of allograft loss after AR. Five case series have described allograft outcome in patients treated with alemtuzumab for AR (1-5). The results of these case series are difficult to compare with our study. Four of the five studies were performed more than 15 years ago and patients in these studies were not treated with the current gold standard therapy (induction therapy in combination with tacrolimus and MMF) (2-5). Furthermore, these case series were of heterogeneous design. First, alemtuzumab was prescribed as first line treatment for $\mathrm{AR}$ in two studies $(4,5)$, and in one study alemtuzumab was prescribed to patients with AR resistant to ATG or OKT3 (2). Second, the dose of alemtuzumab ranged from $15 \mathrm{mg}$ (1) to $93 \mathrm{mg}$ (2). Third, the follow-up period of these studies ranged from 3 months (1) to 10 years (4). Compared with treatment with methylprednisolone or rATG, allograft survival in 


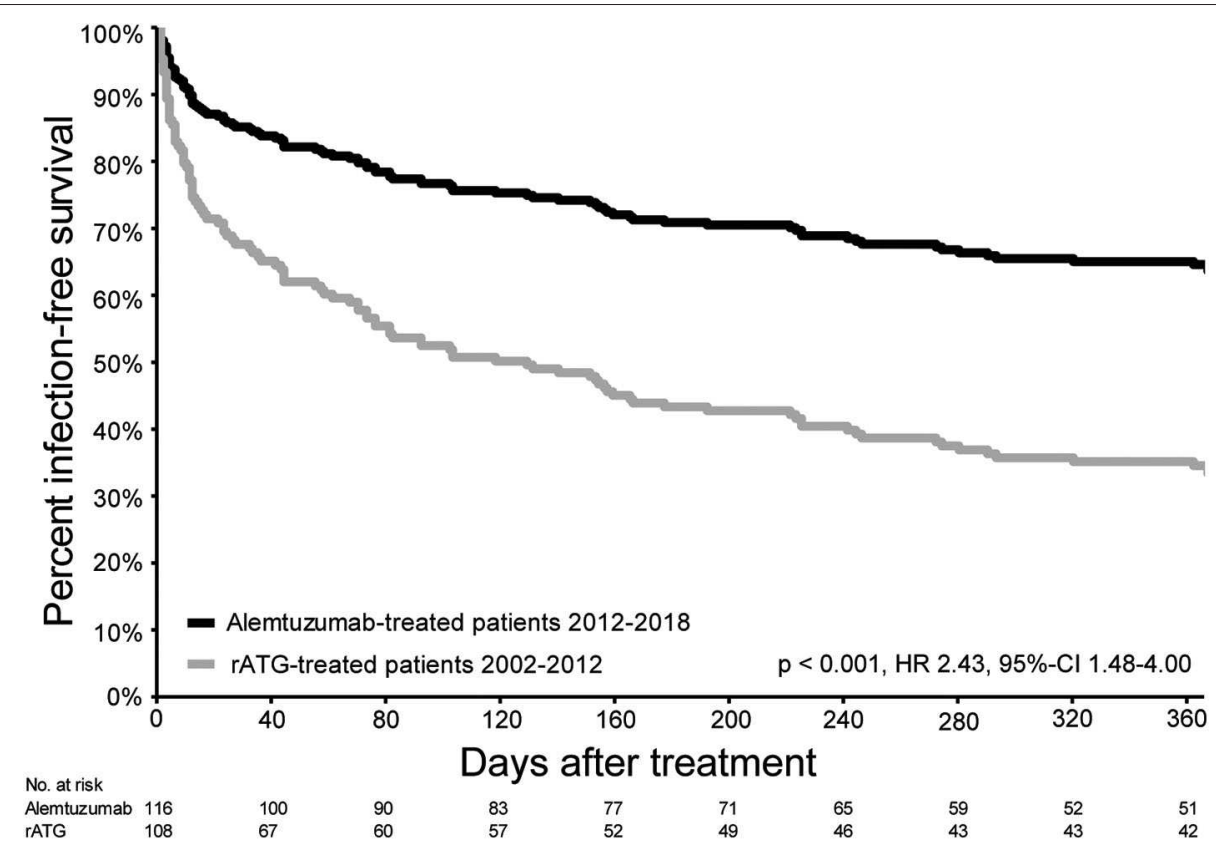

FIGURE 4 | Infection-free survival in the first year after treatment for acute rejection. Infection-free survival (excluding CMV, EBV, and BK virus infections) of patients with AR and treated with alemtuzumab (2012-2018) and patients treated with rATG for AR between 2002 and 2012.

alemtuzumab-treated patients was similar $(1,4)$. The result of our study supports this conclusion.

Treatment with alemtuzumab is associated with serious side effects and therefore the assessment of the benefit-risk balance in the individual patient before initiation of treatment is necessary. We investigated which clinical factors influenced allograft survival. Factors that were associated with a good response were a low $\triangle$ eGFR between baseline and the moment of $\mathrm{AR}$, glucocorticoid maintenance therapy at the time or AR, and an actual PRA below 6\%. The use of glucocorticoid maintenance therapy and timing of rejection were related, because all patients with an early acute rejection used glucocorticoids as maintenance therapy. Therefore, we are not sure if glucocorticoid use is a protective factor, or that an early rejection is associated with a better allograft outcome compared with a late rejection. Late rejections occur in patients who visit the outpatient clinic less frequently and with intervals of 1-4 months, likely leading to a delay in diagnosis.

Surprisingly, patients with more HLA mismatches had a better response to alemtuzumab therapy compared with those with less HLA mismatches. Analysis of all factors showed that patients with more HLA mismatches (4-6) more often received a kidney from a living unrelated donor. How this is related to a better response to alemtuzumab treatment is unclear. It is known that results of living donor kidney transplantation are better compared to deceased donor transplantation, even with higher numbers of HLA mismatches (21). Taken together, based on these results, we treat patients with an early AR aggressively with alemtuzumab and are more reluctant to administer alemtuzumab in patients with a late AR who also have a considerable loss of renal function.

Seventeen patients with aABMR were treated with alemtuzumab. Treatment options for aABMR are limited and no specific drugs have received US Food and Drug Administration approval. Currently, the therapy for aABMR consists of glucocorticoids, IVIg and/or plasma-exchange, although the evidence for this treatment is not strong $(22,23)$. Since alemtuzumab causes lysis of T- and B cells, as well as antigen presenting cells, alemtuzumab may be considered in patients with aABMR. In our study, patients with aABMR showed a good response to alemtuzumab therapy. However, larger studies are necessary to confirm our results and analyze the best therapeutic strategy.

Although $\mathrm{T}$ cell-depletion after alemtuzumab therapy lasts longer than after rATG (24), the infection-free survival was better after therapy with alemtuzumab compared with rATG. The biggest difference in the number of infections in patients treated with rATG or alemtuzumab occurred in the first few weeks after therapy (Table S6). A possible explanation for this is the longer duration of hospitalization after therapy for AR in rATG-treated patients compared with alemtuzumab-treated patients. A longer hospitalization is associated with a higher risk for health care-associated infections (25). The occurrence of CMV disease or reactivation was similar between patients treated with alemtuzumab or rATG. In literature, similar results (lower frequency of infections and no difference in CMV infections) are seen when alemtuzumab or rATG are used as induction therapy (26). 
In contrast to reports investigating the occurrence of autoimmune disorders in patients suffering from multiple sclerosis and treated with alemtuzumab, we observed no clinically apparent autoimmune thyroid disorders or idiopathic thrombocytopenic purpura in the present cohort (27). Possibly, the follow-up period of the present study was too short for these autoimmune events to occur. Another reason could be that patients with multiple sclerosis are susceptible to autoimmune disorders because of their genetic constitution.

The administration of rATG is associated with serious infusion-related side effects and the drug is relatively contraindicated in patients with cardiac failure or fluid overload $(11,12)$. Infusion-related side effects in our study were less prevalent in patients treated with alemtuzumab compared with rATG therapy and subcutaneous administration of alemtuzumab therefore appears to be safe in frail patients and patients with cardiac morbidity. In this study, rATG was given as a bolus of $4 \mathrm{mg} / \mathrm{kg}$. We cannot exclude the possibility that another dosing regimen such as a repeated, standard dose of rATG may have resulted in fewer infusion-related side effects (28).

We acknowledge the limitations of the current study. First, this was a retrospective single-center study. Second, several variables (including time period, the use of induction therapy and others) were different between the patients treated with alemtuzumab and the patients treated with rATG. A propensity score analysis was performed to correct for potential differences between the alemtuzumab and rATG group, but we cannot exclude the possibility that other (unmeasured) confounding factors influenced the outcomes of this analysis. Currently, these data offer the best available evidence for the treatment of AR with alemtuzumab as it is unlikely that a randomized controlled trial comparing alemtuzumab with other anti-rejection therapies will be performed anytime soon. Third, the allograft survival of patients who were treated with alemtuzumab seemed (although not significant) to be worse compared with rATG-treated patients. Again, we cannot exclude the possibility that inclusion of more patients may have resulted in a significant difference between the two groups. Fourth, in our study $93.8 \%$ of alemtuzumab-treated patients were treated with basiliximab induction therapy. In the United States, only 33.8\% of kidney transplant recipients are treated with basiliximab, whereas $65.9 \%$ of patients receive induction therapy with $\mathrm{T}$ cell-depleting antibodies (29). We don't know the influence of this difference on the outcomes after alemtuzumab therapy for AR. Fifth, due the unavailability of data on DSAs in the rATG-treated patients, it was not possible to apply the Banff 2017 classification on biopsies of these patients which may have biased the diagnosis of ABMR.

To conclude, alemtuzumab therapy could be an alternative therapy to rATG for glucocorticoid-resistant or severe AR. This may be especially relevant for patients with a relative contraindication for rATG, including patients suffering from fluid overload or previous rATG treatment. Further studies, preferably multicenter randomized controlled trials, are necessary to explore the potential advantages of alemtuzumab for severe rejection.

\section{DATA AVAILABILITY STATEMENT}

The datasets generated for this study are available on request to the corresponding author.

\section{ETHICS STATEMENT}

The studies involving human participants were reviewed and approved by Erasmus MC ethical board. Written informed consent for participation was not required for this study in accordance with the national legislation and the institutional requirements.

\section{AUTHOR CONTRIBUTIONS}

MZ participated in the research design, acquisition of the data, data analysis, and writing of the article. MC-V participated in the revision of the Banff classification of the kidney biopsies and critical revision of the manuscript. DR acquired the data of donor specific anti-HLA antibodies. KM and JR participated in data analysis and critical revision of the manuscript. $\mathrm{MK}, \mathrm{MH}, \mathrm{MA}$, and $\mathrm{CB}$ participated in the critical revision of the manuscript. $\mathrm{DH}$ participated in the research design, data analysis, and critical revision of the manuscript. All authors contributed to the article and approved the submitted version.

\section{ACKNOWLEDGMENTS}

The authors thank J. Kal-Van Gestel and J. J. M Hassink for assistance with data retrieval.

\section{SUPPLEMENTARY MATERIAL}

The Supplementary Material for this article can be found online at: https://www.frontiersin.org/articles/10.3389/fimmu. 2020.01332/full\#supplementary-material

Figure S1 | Survival plots of allograft- and patient survival in the period between 2002 and 2012 and 2012-2018. (A) Kaplan-Meier patient survival curve of patients treated with alemtuzumab for AR (2012-2018) and patients transplanted in the same period and not treated with alemtuzumab. From time point of acute rejection (alemtuzumab group) and time point of kidney transplantation (patients not treated with alemtuzumab). (B) Kaplan-Meier allograft survival curve (event = allograft loss, censored for death) of patients treated with alemtuzumab for AR (2012-2018) and patients transplanted in the same period and not treated with alemtuzumab. From time point of acute rejection (alemtuzumab group) and time point of kidney transplantation (patients not treated with alemtuzumab). (C) Kaplan-Meier patient survival curve of patients treated with rATG for AR (2002-2012) and patients transplanted in the same period and not treated with rATG. From time point of acute rejection (rATG group) and time point of kidney transplantation (patients not treated with rATG). (D) Kaplan-Meier allograft survival curve (event = allograft loss, censored for death) of patients treated with rATG for AR (2002-2012) and patients transplanted in the same period and not treated with rATG. From time point of acute rejection (rATG group) and time point of kidney transplantation (patients not treated with rATG).

Figure S2 | Kaplan-Meier survival curves of patient survival of different age categories. (A) Patient survival of patients ( $<50$ years at time of transplantation) treated with alemtuzumab for AR (2012-2018) and patients $(<50$ years at time of transplantation) transplanted in the same period and not treated with alemtuzumab. (B) Patient survival of patients (50-65 years at time of transplantation) treated with alemtuzumab for AR (2012-2018) and patients 
(50-65 years at time of transplantation) transplanted in the same period and not treated with alemtuzumab. (C) Patient survival of patients ( $>65$ years at time of transplantation) treated with alemtuzumab for AR (2012-2018) and patients (>65 years at time of transplantation) transplanted in the same period and not treated with alemtuzumab.

Figure S3 | The creatinine clearance $\left(\mathrm{mL} / \mathrm{min} / 1.73 \mathrm{~m}^{2}\right)$ of patients treated with alemtuzumab (A) or rATG (B) for AR. The boxes represent median and IQR and the whiskers 5th and 95th percentile. N, number of patients with an eGFR; Baseline, best serum creatinine or eGFR in 3 month before AR; 0 , serum creatinine or eGFR on day of AR; M3, 3 months after alemtuzumab or rATG $( \pm 4$ weeks); M6, 6 months ( \pm 6 weeks) after alemtuzumab or rATG; M12, 12 months after alemtuzumab or $\mathrm{rATG}\left( \pm 8\right.$ weeks). ${ }^{*} p<0.05,{ }^{* *} p=$ not significant.

Figure S4 | Kaplan-Meier survival curve of allograft survival of alemtuzumab-treated patients with aTCMR or aABMR.

Figure S5 | T- and B cells after alemtuzumab therapy. T- and B cells were measured every 3 months, until T cells were $>200 \times 10^{6} / \mathrm{L}$. (A) Scatter dot plot of all measured $T$ cells on different time points after alemtuzumab therapy. The horizontal line depicts the median. (B) Percent of patients with repopulation of $\mathrm{T}$

\section{REFERENCES}

1. van den Hoogen MW, Hesselink DA, van Son WJ, Weimar W, Hilbrands LB. Treatment of steroid-resistant acute renal allograft rejection with alemtuzumab. Am J Transplant. (2013) 13:192-6. doi: 10.1111/j.1600-6143.2012.04328.x

2. Csapo Z, Benavides-Viveros C, Podder H, Pollard V, Kahan BD. Campath- $1 \mathrm{H}$ as rescue therapy for the treatment of acute rejection in kidney transplant patients. Transplant Proc. (2005) 37:2032-6. doi: 10.1016/j.transproceed.2005.03.042

3. Basu A, Ramkumar M, Tan HP, Khan A, McCauley J, Marcos A, et al. Reversal of acute cellular rejection after renal transplantation with Campath-1H. Transplant Proc. (2005) 37:923-6. doi: 10.1016/j.transproceed.2004.12.019

4. Clatworthy MR, Friend PJ, Calne RY, Rebello PRU, Hale G, Waldmann H, et al. Alemtuzumab (CAMPATH-1H) for the treatment of acute rejection in kidney transplant recipients: long-term follow-up. Transplantation. (2009) 87:1092-5. doi: 10.1097/TP.0b013e31819d3353

5. Rebello PR, Hale G, Friend PJ, Cobbold SP, Waldmann H. Antiglobulin responses to rat and humanized CAMPATH-1 monoclonal antibody used to treat transplant rejection. Transplantation. (1999) 68:141720. doi: 10.1097/00007890-199911150-00032

6. van der Zwan M, Baan CC, van Gelder T, Hesselink DA. Review of the clinical pharmacokinetics and pharmacodynamics of alemtuzumab and its use in kidney transplantation. Clin Pharmacokinet. (2018) 57:191207. doi: 10.1007/s40262-017-0573-x

7. Genzyme Submits Applications to FDA and EMA for Approval of LEMTRADA ${ }^{T M}$ (alemtuzumab) for Multiple Sclerosis. (2012). Available online at: http://news.genzyme.com/press-release/genzyme-submits-applicationsfda-and-ema-approval-lemtrada-alemtuzumab-multiple-sclero (accessed June 12, 2012).

8. Campath Distribution Program. Available online at: http://www.campath. $\mathrm{com} /$ (accessed April 12, 2019).

9. Tai E, Chapman JR. The KDIGO review of the care of renal transplant recipient. Pol Arch Med Wewn. (2010) 120:237-42. doi: 10.20452/pamw.935

10. van der Zwan M, Clahsen-Van Groningen MC, Roodnat JI, Bouvy A, Slachmuylders CL, Weimar W, et al. The efficacy of rabbit anti-thymocyte globulin for acute kidney transplant rejection in patients using calcineurin inhibitor and mycophenolate mofetil-based immunosuppressive therapy. Ann Transplant. (2018) 23:577-90. doi: 10.12659/AOT.909646

11. Buchler M, Hurault de Ligny B, Madec C, Lebranchu Y, French Thymoglobuline Pharmacovigilance Study G. Induction therapy by anti-thymocyte globulin (rabbit) in renal transplantation: a 1-yr follow-up of safety and efficacy. Clin Transplant. (2003) 17:539-45. doi: 10.1046/j.1399-0012.2003.00102.x cells $>200 \times 10^{6} / \mathrm{L}$ in the year after alemtuzumab therapy. (C) Scatter dot plot of all measured $B$ cells on different time points after alemtuzumab therapy. The horizontal line depicts the median. (D) Percent of patients with repopulation of $B$ cells $>100 \times 10^{6} / \mathrm{L}$ in the year after alemtuzumab therapy.

Table S1 | Patients with a second biopsy between methylprednisolone and alemtuzumab to confirm ongoing rejection.

Table S2 | Cause of death after therapy with alemtuzumab or rATG.

Table S3 | Univariable Cox proportional hazard regression analysis for risk of death within patients treated with alemtuzumab.

Table S4 | Univariable Cox proportional hazard regression analysis for allograft loss in patients treated with alemtuzumab.

Table S5 | Characteristics and statistical analysis of alemtuzumab-treated patients with HLA mismatch of 0-3, and patients with HレA mismatch of 4-6.

Table S6 | Infections during the total follow-up after alemtuzumab and rATG treatment.

Table S7 | Malignancies after alemtuzumab treatment.

Table S8 | Malignancies after rATG treatment.

12. Tanriover B, Chuang P, Fishbach B, Helderman JH, Kizilisik T, Nylander W, et al. Polyclonal antibody-induced serum sickness in renal transplant recipients: treatment with therapeutic plasma exchange. Transplantation. (2005) 80:27981. doi: 10.1097/01.TP.0000165093.13046.B3

13. van Agteren $\mathrm{M}$, Weimar $\mathrm{W}$, de Weerd $\mathrm{AE}$, Te Boekhorst PAW, Ijzermans JNM, van de Wetering J, et al. The first fifty ABO blood group incompatible kidney transplantations: the rotterdam experience. J Transplant. (2014) 2014:913902. doi: 10.1155/2014/913902

14. Haas M, Loupy A, Lefaucheur C, Roufosse C, Glotz D, Seron D, et al. The Banff 2017 Kidney meeting report: revised diagnostic criteria for chronic active $\mathrm{T}$ cell-mediated rejection, antibody-mediated rejection, and prospects for integrative endpoints for next-generation clinical trials. Am J Transplant. (2018) 18:293-307. doi: 10.1111/ajt.14625

15. Roufosse C, Simmonds N, Clahsen-van Groningen M, Haas M, Henriksen KJ, Horsfield C, et al. Reference guide to the banff classification of renal allograft pathology. Transplantation. (2018) 102:1795-814. doi: 10.1097/TP.0000000000002366

16. Loupy A, Haas M, Solez K, Racusen L, Glotz D, Seronet D, et al. The Banff 2015 kidney meeting report: current challenges in rejection classification and prospects for adopting molecular pathology. Am J Transplant. (2017) 17:28-41. doi: 10.1111/ajt.14107

17. Levey AS, Stevens LA, Schmid CH, Schmis CH, Zhang YL, Castro AF, et al. A new equation to estimate glomerular filtration rate. Ann Intern Med. (2009) 150:604-12. doi: 10.7326/0003-4819-150-9-200905050-00006

18. Clatworthy MR, Sivaprakasam R, Butler AJ, Watson CJE. Subcutaneous administration of alemtuzumab in simultaneous pancreas-kidney transplantation. Transplantation. (2007) 84:15637. doi: 10.1097/01.tp.0000295718.55669.3a

19. Using Propensity Scores With Small Samples. Available online at: http://www. faculty.umb.edu/william_holmes/usingpropensityscoreswithsmallsamples. pdf (accessed June 3, 2019).

20. van der Zwan $M$, Hesselink DA, Brusse $E$, van Doorn $P A$, van den Hoogen MWF, de Weerd AE, et al. Guillain-Barre syndrome and chronic inflammatory demyelinating polyradiculoneuropathy after alemtuzumab therapy in kidney transplant recipients. Neurol Neuroimmunol Neuroinflamm. (2020) 7:e721. doi: 10.1212/NXI.0000000000000721

21. Laging M, Kal-van Gestel JA, Haasnoot GW, Class FHJ, van de Wetering J, Ijzermans JNM, et al. Transplantation results of completely HLA-mismatched living and completely HLA-matched deceased-donor kidneys are comparable. Transplantation. (2014) 97:330-6. doi: 10.1097/01.TP.0000435703.61642.43

22. Archdeacon P, Chan M, Neuland C, Velidedeoglu E, Meyer J, Tracy L, et al. Summary of FDA antibody-mediated rejection workshop. Am J Transplant. (2011) 11:896-906. doi: 10.1111/j.1600-6143.2011. 03525.x 
23. Sablik KA, Clahsen-van Groningen MC, Looman CWN, Damman J, van Agteren M, Betjes MGH. Treatment with intravenous immunoglobulins and methylprednisolone may significantly decrease loss of renal function in chronic-active antibody-mediated rejection. BMC Nephrol. (2019) 20:218. doi: 10.1186/s12882-019-1385-z

24. Product Monograph of Thymoglobulin. Available online at: http://products. sanofi.ca/en/thymoglobulin.pdf (accessed May 24, 2019).

25. Leape LL, Brennan TA, Laird N, Lawthers AG, Localio AR, Barnes BA, et al. The nature of adverse events in hospitalized patients. Results of the harvard medical practice study II. $N$ Engl J Med. (1991) 324:377-84. doi: 10.1056/NEJM1991020732 40605

26. Hanaway MJ, Woodle ES, Mulgaonkar S, Peddi VR, Kaufman DB, First MR, et al. Alemtuzumab induction in renal transplantation. N Engl J Med. (2011) 364:1909-9. doi: 10.1056/NEJMoa1009546

27. Ziemssen T, Thomas K. Alemtuzumab in the long-term treatment of relapsing-remitting multiple sclerosis: an update on the clinical trial evidence and data from the real world. Ther Adv Neurol Disord. (2017) 10:34359. doi: 10.1177/1756285617722706

28. Kho MM, Bouvy AP, Cadogan M, Kraaijeveld R, Baan CC, Weimear WA. The effect of low and ultra-low dosages Thymoglobulin on peripheral $\mathrm{T}$, B and NK cells in kidney transplant recipients. Transpl Immunol. (2012) 26:186-90. doi: 10.1016/j.trim.2012.02.003
29. Hart A, Smith JM, Skeans MA, Gustafson SK, Wilk AR, Castro S, et al. OPTN/SRTR 2017. Annual data report: kidney. Am J Transplant. (2019) 19(Suppl. 2):19-123. doi: 10.1111/ajt.15274

Conflict of Interest: DH has received grant support, lecture, and consulting fees from Astellas Pharma and Chiesi Pharmaceuticals, as well as a lecture fee from Hikma Pharma and grand support from Bristol Myers-Squibb. MC-V has received grant support from Astellas Pharma. $\mathrm{MH}$ has received grant support from Novartis and Shire and lecture fees from Astellas Pharma, Chiesi Pharmaceuticals, MSD, Sanofi/Genzyme, Shire, and Vifor Pharma.

The remaining authors declare that the research was conducted in the absence of any commercial or financial relationships that could be construed as a potential conflict of interest.

Copyright (C) 2020 van der Zwan, Clahsen-Van Groningen, van den Hoogen, Kho, Roodnat, Mauff, Roelen, van Agteren, Baan and Hesselink. This is an open-access article distributed under the terms of the Creative Commons Attribution License (CC $B Y)$. The use, distribution or reproduction in other forums is permitted, provided the original author(s) and the copyright owner(s) are credited and that the original publication in this journal is cited, in accordance with accepted academic practice. No use, distribution or reproduction is permitted which does not comply with these terms. 